\title{
Optimal Open-Loop Routing and Threshold-Based Allocation in TWO Parallel QUEUEING Systems with Heterogeneous Servers
}

\author{
Dmitry Efrosinin $1,2, *\left(\mathbb{D}\right.$ and Natalia Stepanova ${ }^{3}$ (D) \\ 1 Institute for Stochastics, Johannes Kepler University Linz, 4040 Linz, Austria \\ 2 Department of Information Technologies, Faculty of Mathematics and Natural Sciences, Peoples' Friendship \\ University of Russia (RUDN University), 117198 Moscow, Russia \\ 3 Laboratory 17, V.A. Trapeznikov Institute of Control Sciences of RAS, 117997 Moscow, Russia; \\ natalia0410@rambler.ru \\ * Correspondence: dmitry.efrosinin@jku.at
}

Citation: Efrosinin, D.; Stepanova, N. Optimal Open-Loop Routing and Threshold-Based Allocation in TWO Parallel QUEUEING Systems with Heterogeneous Servers. Mathematics 2021, 9, 2766. https://doi.org/ $10.3390 /$ math 9212766

Academic Editor: Juan Ramón Torregrosa Sánchez

Received: 21 September 2021 Accepted: 25 October 2021 Published: 31 October 2021

Publisher's Note: MDPI stays neutral with regard to jurisdictional claims in published maps and institutional affiliations.

Copyright: (c) 2021 by the authors. Licensee MDPI, Basel, Switzerland. This article is an open access article distributed under the terms and conditions of the Creative Commons Attribution (CC BY) license (https:// creativecommons.org/licenses/by/ $4.0 /)$.

\begin{abstract}
In this paper, we study the problem of optimal routing for the pair of two-server heterogeneous queues operating in parallel and subsequent optimal allocation of customers between the servers in each queue. Heterogeneity implies different servers in terms of speed of service. An open-loop control assumes the static resource allocation when a router has no information about the state of the system. We discuss here the algorithm to calculate the optimal routing policy based on specially constructed Markov-modulated Poisson processes. As an alternative static policy, we consider an optimal Bernoulli splitting which prescribes the optimal allocation probabilities. Then, we show that the optimal allocation policy between the servers within each queue is of threshold type with threshold levels depending on the queue length and phase of an arrival process. This dependence can be neglected by using a heuristic threshold policy. A number of illustrative examples show interesting properties of the systems operating under the introduced policies and their performance characteristics.
\end{abstract}

Keywords: parallel queues; open-loop policy; Markov decision process; threshold policy; matrixanalytic approach; difference equations

\section{Introduction}

The very rapidly spreading and upgrading of telecommunications and computer technology has led to combination and simultaneous maintenance of inter-generational systems. Various hybrid and heterogeneous service systems, where the servers are differentiated by their service rates, usage costs or reliability attributes, are being created to ensure proper quality and reliability of service while wishing to ensure energy efficiency and generally limit the amount of resources used. In many practical service applications it has become necessary to provide stochastic modeling by means of queueing systems consisting of individual queues and operating in parallel. Such systems often face the challenge of optimal routing between parallel queues which can be either dynamic (also called closed-loop policy) or static (open-loop policy). In the first case, the router dynamically receives information about the state of the system, while in the second case, the routing policy is based only on the information available at the initial point in time.

Most of papers dedicated to the parallel queueing systems deal with a dynamic routing. The homogeneous case, where each parallel queue is supplied with only one server and all of them are identical, was studied in [1]. It was shown that the shortest queue (SQ) policy is optimal for the average cost criterion. A similar heterogeneous server model was analyzed in [2]. The authors proved the result that the policy to use the faster server with a shorter queue minimizes the expected cost. In [3], a dynamic programming approach was used 
to find an optimal routing policy with the aim to minimize the expected total discounted cost. It was shown that the optimal policy has a switch structure. The optimal routing problems in finite-source parallel queueing system were presented in [4,5]. A classical routing problem in exponential system with $K$ parallel heterogeneous servers was studied in [6] with respect to the SQ policy which, in general, eliminate the service rates and cost structure and to the shortest expected delay (SED) routing which takes these attributes into account. Systems where each of the parallel queues is supplied with a multi-server service area were considered in [7], where a static policy in form of the optimal Bernoulli splitting, and three alternative dynamic index policies were considered. Obviously, when system information is available to the router during the decision-making process, it can respond more flexibly to changing system states, which means lower values for the optimised mean cost function as a result. Unfortunately, in certain cases, the transmission of such information to the router is not always reliable and control errors can occur taking into account possible losses and delays. Alternatively an open-loop control policy can be used instead. Ref. [8] considered the problem of routing $N$ arrivals to $M$ queues in parallel without information about system states and proposed two policy iteration algorithms. The optimal routing for a static policy based on a routing words was investigated in [9]. The authors proposed some heuristics used to approximate the optimal policy.

The main aim of the paper is to study the open-loop control problem with an application to parallel queues, each of them has two heterogeneous servers operating at different service rates. In contrast to the previous research, this model is new one, since the optimal routing problem must be solved simultaneously both for the routing between the queues and for the allocation between the servers within each queue. Furthermore, the control policy for the routing between queues must be of an open-loop type, i.e., be independent of the state changing, and the control allocation policy for the servers should depend only on the number of customers in a certain queue. Our analysis of the proposed system includes the following contributions. We define the arrival process to each queue as a special case of a Markov-modulated Poisson process (MMPP) with $l$ phases, where the transition within a set of the first $k$ phases in the first queue and $l-k$ phases in the second queue, $1 \leq k \leq l$, accompany the arrival of a new customer. For the fixed parameters $l$ and $k$ we formulate the Markov decision problem to calculate the optimal control policy for the allocation of customers between the servers to minimize the long-run average cost per unit of time. Then, the model is optimized with respect to parameters $k$ and $l$. We show that the dynamic-programming value function satisfies certain monotonicity properties indicating that the optimal allocation policy is of the threshold type with threshold levels depending on the queue length and the phase of the arrival process. Further, we propose a heuristic threshold policy depending only on the queue length and derive a matrix-analytic solution for the mean number of customers in the system. As an alternative model with a static policy we consider an optimal Bernoulli splitting policy and obtain a closed-form solution for the stationary state probabilities and the optimized functional.

The rest of the paper is organized as follows. Section 2 formally describes the queueing model where the optimal routing between the queues is formulated as an optimal number of phases in MMPP arrival process connected with arrivals and provides the MDP formulation for the optimal allocation between servers for a certain queue. Section 3 presents the results required to show the optimality of a threshold allocation policy and a corresponding matrix-analytic solution under a heuristic threshold policy. The Bernoulli splitting policy is discussed in Section 4. A brief review for the proposed results is given in Section 5.

For use in sequel, let $\mathbf{e}(n), \mathbf{e}_{j}(n)$ and $I_{n}$ denote, respectively, the $n$-dimensional unit vector, the unit vector with each element equal to zero except $j$ th unity element, an identity matrix of dimension $n$. When there is no need to specify the dimension of these vectors we will omit the corresponding argument. The notation ${ }^{\top}$ appearing in a vector or in a matrix stand for the matrix transpose. Denote further by $H_{i, j}$ the square matrix of the suitable dimension where only the element in the $i$ th row and $j$ th column is 1 and 0 elsewhere. The 
notation $\otimes$ will stand for the Kronecker product of two matrices. Denote by $S_{j}, j \geq 1$, the shift operators, i.e., $S_{j} x=x+\mathbf{e}_{j}$ and $S_{j}^{n} x=x+n \mathbf{e}_{j}$ for $n \geq 2$ applied to a vector $x$.

\section{Routing with an Open-Loop Control and MDP Formulation}

The queueing system shown in Figure 1 is studied. This system consists of two exponential parallel queues: $Q_{1}$-Queue 1 and $Q_{2}$-Queue 2. The arrival stream forms a Poisson process with a rate $\lambda$. Each queue is supplied with two heterogeneous servers operating without pre-emption, i.e., the customer is not able to change the server during a service time. The service rates of the queue $Q_{i}$ are $\mu_{1 i}$ and $\mu_{2 i}$ with $\mu_{1 i}>\mu_{2 i}$. We assume that the first queue has a faster service area as the second one, i.e., $\mu_{11}>\mu_{12}$ and $\mu_{21}>\mu_{22}$. The Router 0 allocates the customers between the queues while the Routers 1 and 2 assign the customers between the servers of the corresponding queue. The main task consists both in optimal open-loop routing of customers to parallel queues and in their optimal allocation between heterogeneous servers within each queue. As it is known, see e.g., [10-13], in an ordinary queueing system with two heterogeneous servers operating without pre-emption, one common waiting line and a Poisson arrival stream the optimal allocation policy has a threshold structure, where the fastest server must be occupied whenever it is idle and slower server is used according to a specified threshold level for the queue length. We have to find out if the optimal policy for the allocation between the servers in the system under study exhibits similar structural properties for non-Poisson arrival stream. In this case we could have a static control not only for the routing between the queues but also for the servers.

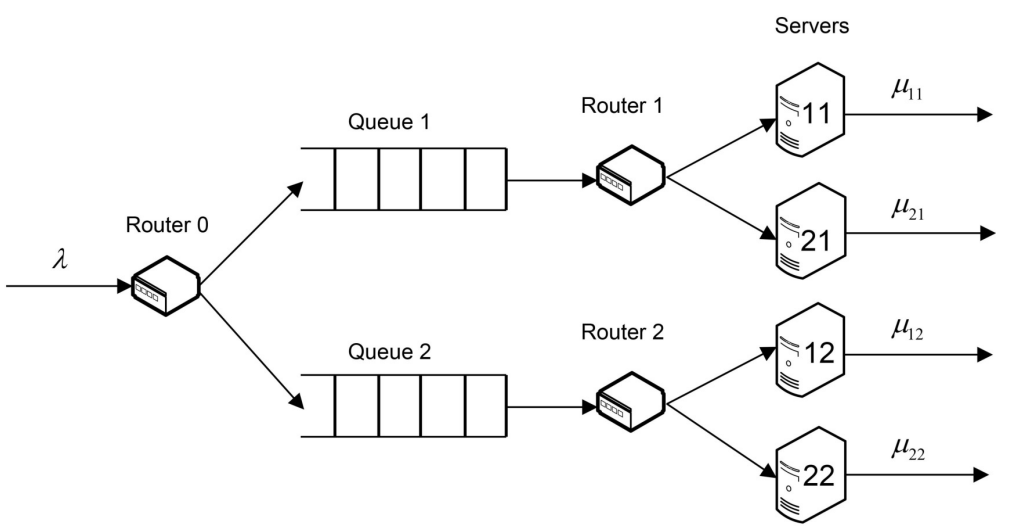

Figure 1. Controllable multi-server queueing system with heterogeneous servers and operating costs.

This section will look at the situation where separate queues receive applications according to the open-loop control when the information for the Router 0 is available at starting time point. We define the routing policy as a periodic binary sequence $\{0,1\}^{l}$ with a period length $l$, where 1 means that the customer is sent to $Q_{1}$ and 0 - the customer is sent to $Q_{2}$. Note that for the routing policy $f_{0}$ in stationary regime only the number $k \leq l$ of $1 \mathrm{~s}$ in a binary sequence of length $l$ for one queue matters but not their specific locations. In other words, if $k$ is the number of $1 \mathrm{~s}$ for the routing to the first queue, then $l-k$ is the number of $1 \mathrm{~s}$ for the routing to the second queue. Denote by $u(i)=(l-2 k)(i-1)+k, i=1,2$. For the arbitrary sequence a parallel queueing system $Q_{i}$ behaves as a $G_{i} / M_{i} / 2$ queue with two heterogeneous servers. For a given routing policy $f_{0}=k$ the arrival process in the queue $Q_{i}$ can be formulated as a special case of a Markov-modulated Poisson process (MMPP). This arrival process is defined by two square matrices $D_{0 i}$ and $D_{1 i}$ of dimension $l$, 


$$
D_{0 i}=\left(\begin{array}{ccccccc}
-\lambda & 0 & 0 & 0 & 0 & \ldots & 0 \\
0 & -\lambda & 0 & 0 & 0 & \ldots & 0 \\
\ddots & \ddots & \ddots & \ddots & \ddots & \ldots & \vdots \\
0 & \ldots & -\lambda & 0 & 0 & \ldots & 0 \\
0 & \ldots & 0 & -\lambda & \lambda & \ldots & 0 \\
\ddots & \ddots & \ddots & \ddots & \ddots & \ldots & \vdots \\
0 & \ldots & 0 & 0 & \ldots & -\lambda & \lambda \\
\lambda & \ldots & 0 & 0 & \ldots & 0 & -\lambda
\end{array}\right), \quad D_{1 i}=\left(\begin{array}{ccccccc}
0 & \lambda & 0 & 0 & 0 & \ldots & 0 \\
0 & 0 & \lambda & 0 & 0 & \ldots & 0 \\
\ddots & \ddots & \ddots & \ddots & \ddots & \ldots & \vdots \\
0 & \ldots & 0 & 0 & \lambda & \ldots & 0 \\
0 & \ldots & 0 & 0 & 0 & \ldots & 0 \\
\ddots & \ddots & \ddots & \ddots & \ddots & \ldots & \vdots \\
0 & \ldots & 0 & 0 & 0 & \ldots & 0 \\
0 & \ldots & 0 & 0 & 0 & \ldots & 0
\end{array}\right)
$$

Here, $D_{i}=D_{0 i}+D_{1 i}$ is a generator of the continuous-time Markov chain associated with an arrival process with a state space $\{1,2, \ldots, l\}$, i.e., $D_{i} \mathbf{e}(l)=\mathbf{0}$. The matrix $D_{0 i}$ contains transitions without arrivals while $D_{1 i}$ includes transition rates accompanying with a new arrival. The average arrival rate $\bar{\lambda}_{i}=\boldsymbol{\alpha}_{i} D_{1 i} \mathbf{e}(l)=\frac{u(i)}{l} \lambda$, where $\boldsymbol{\alpha}_{i}=\frac{1}{l} \mathbf{e}^{\top}(l)$ is a solution of a linear system $\boldsymbol{\alpha}_{i} D_{i}=\mathbf{0}$ and $\boldsymbol{\alpha}_{i} \mathbf{e}(l)=1$. The lag-1 correlation coefficient $r_{i}$ of the inter-arrival times in the queue $Q_{i}$ is computed by

$$
r_{i}=\frac{\lambda u(i) \mathbf{e}^{\top}(l)\left(-D_{0 i}\right)^{-1} D_{1 i}\left(-D_{0 i}\right)^{-1} \mathbf{e}(l)-l^{2}}{2 \bar{\lambda} \mathbf{e}^{\top}(l)\left(-D_{0}\right)^{-1} \mathbf{e}(l)-l^{2}} .
$$

Further, in the paper we use the fact that in each queue $Q_{1}$ and $Q_{2}$ the faster server must always be used as was shown for Poisson arrivals in [11,12] and phase type arrival processes in $[14,15]$. Therefore, we collapse the state space to three dimensions avoiding a state specification for the faster server. Due to capacity limits and the fact that proof is similar to that in the references above, we skip it. Denote by $Q_{i}(t), S_{i}(t)$, and $N_{i}(t)$, respectively the total number of waiting customers and at the fast server, the number of customers at the second server and the state of an arrival process at time $t$ in a certain queue $Q_{i}$. The Markov decision process is associated with a continuous-time Markov-chain

$$
\left\{X_{i}(t)\right\}_{t \geq 0}=\left\{Q_{i}(t), S_{i}(t), N_{i}(t)\right\}_{t \geq 0}
$$

with a state space given by

$$
E=\{x=(q, s, n): q \geq 0, s \in\{0,1\}, j=1,2,1 \leq n \leq l\} .
$$

Denote by $q(x), d_{j}(x)$ and $n(x)$ the corresponding components of the vector state $x \in E$. The decision epochs consists of the moments when the change of an arrival state is accompanied with a real arrival which occurs if $1 \leq n(x) \leq k$ and service completions while the queue is non empty. The action space is $A=\{0,1,2\}$ where as usual $a=0$ means to put a customer to the queue and $a \neq 0$ means to assign a customer to the $a$ th server and $A(x) \subseteq A$-the subsets of actions in state $x \in E$. The immediate cost $c(x)$ is the number of customers in state $x$, i.e., $c(x)=q(x)+d_{1}(x)+d_{2}(x)$. The optimization problem consists to find the optimal policy $f=\left\{k, f_{i}^{u(i)}, i=1,2\right\}$ including the routing policy $f_{0}=k$ and the corresponding allocation policies $f_{1}^{k}$ and $f_{2}^{l-k}$ to minimize the long-run average number of customers in the system $\bar{N}^{f}=g^{f}$, where

$$
\min _{f} g^{f}=\min _{k, f_{1}^{k}, f_{2}^{l-k}} \sum_{i=1}^{2} g_{i}^{u(i)}
$$

and

$$
g_{i}^{f_{i}^{u(i)}}=\limsup _{t \rightarrow \infty} \frac{1}{t} \mathbb{E}_{i}^{f_{i}^{u(i)}}\left[\int_{0}^{t}\left(Q_{i}(t)+D_{i}(t)\right) d t \mid X_{i}(0)=x_{0}\right], i=1,2
$$


Consider the $i$ th queue of the original system. The optimality equation of the MDP model for such a queue can be expressed using uniformization in the form

$$
\begin{aligned}
v_{i}(x) & =\frac{1}{L_{i}}\left[c(x)-g_{i}+\lambda T v_{i}\left(S_{3}^{m(x)} x\right) 1_{\{n(x) \leq k\}}+\lambda v_{i}\left(S_{3}^{m(x)} x\right) 1_{\{n(x)>k\}}\right. \\
& +\mu_{1 i}\left(v_{i}(x) 1_{\{q(x)=0\}}+v_{i}\left(S_{1}^{-1} x\right) 1_{\{q(x)>0\}}\right) \\
& +\mu_{2 i}\left(v_{i}(x) 1_{\{d(x)=0, q(x)=0\}}+T v_{i}\left(S_{1}^{-1} x\right) 1_{\{d(x)=0, q(x)>0\}}\right. \\
& \left.+v_{i}\left(S_{2}^{-1} x\right) 1_{\{d(x)=1, q(x) \leq 1\}}+T v_{i}\left(S_{1}^{-1} S_{2}^{-1} x\right) 1_{\{d(x)=1, q(x)>1\}}\right]=B v_{i}(x),
\end{aligned}
$$

where $B$ is a dynamic-programming operator, $v_{i}: E \rightarrow \mathbb{R}$ is a relative value function, and $\operatorname{Tv}_{i}(x)=\min _{a \in A(x)} v_{i}\left(S_{a} x\right), x \in E$, is an event-based operator, $m(x)=1+(n(x) \bmod l)-$ $n(x)$ and $L_{i}=\lambda+\mu_{1 i}+\mu_{2 i}$. The second and third terms in the right-hand side of (3) describe the changing of the phase in the arrival process, respectively, accompanied with an arrival of a new customer and without an arrival. The fourth and fifth term stand for service completions, respectively, at server 1 and 2 . Note that the terms including $v_{i}(x)$ for $q(x)=0$ and the term $T v_{i}\left(S_{1}^{-1} x\right)$ for $q(x)>0$ represent fictitious transitions in accordance with the uniformization procedure, as shown in [16]. In the latter case, we allow the action to be performed which is required for the proof of the monotonicity properties. However, as it was discussed in [12], the optimal policy in a system with decision actions at fictitious transition epochs remains the same as in the original system without such actions. In general case the MDP model is assumed to be countable infinite so the cost in a state can not be bounded. To prove the existence of the stationary average-cost optimal policy and convergence of the policy-iteration algorithm $[16,17]$ in this case it is necessary to use the main theorem proposed in [18] by checking the Assumptions 1, 2, 3, and 3*.

Further, we want to show that in each queue $Q_{i}$ there exists an optimal threshold policy that depends on the queue length and the state of an arrival process. To complete it, it is required to study some properties of the function $v(x)$. Define a real-valued function $v: E \rightarrow \mathbb{R}$. We say that $v \in \mathcal{F}_{1}$ if the following monotonicity inequalities hold:

$$
\begin{aligned}
& v(x) \leq v\left(S_{1} x\right), x \in E, \\
& v(x) \leq v\left(S_{2} x\right), d(x)=0, x \in E .
\end{aligned}
$$

Proposition 1. If $v \in \mathcal{F}_{1}$, then for the operator $B$ defined in (3) holds $B v \in \mathcal{F}_{1}$.

Proof. The fact that the operator $T$, as well as $B$ preserves the properties (4) and (5) can be proved in the same way as it has been done in $[12,15]$ for the ordinary infinite population heterogeneous queueing system, therefore the details will be omitted.

Consider now the real valued function $v \in \mathcal{F}_{1}$ which additionally belongs to a set $\mathcal{F}_{2}$ when the monotonicity properties of increments

$$
\begin{aligned}
& v\left(S_{1} x\right)-v\left(S_{2} x\right)-v\left(S_{1}^{2} x\right)+v\left(S_{1} S_{2} x\right) \leq 0, x \in E, d(x)=0, \\
& v\left(S_{1} x\right)-v(x)-v\left(S_{1} S_{2} x\right)+v\left(S_{2} x\right) \leq 0, x \in E, d(x)=0, \\
& 2 v\left(S_{1} x\right)-v(x)-v\left(S_{1}^{2} x\right) \leq 0, x \in E .
\end{aligned}
$$

The superconvexity condition (6) confirms optimality of a threshold-based policy for the usage of second server. To prove it we need to use a supermodularity condition (7) together with a convexity condition (8), which can be obtained by summing (6) and (7).

Proposition 2. If $v \in \mathcal{F}_{2}$, then for the operator $B$ defined in (3) holds $B v \in \mathcal{F}_{2}$. 
Proof. For the operator $B$ according to (3) we obtain

$$
\begin{aligned}
& B v\left(S_{1} x\right)-B v\left(S_{2} x\right)-B v\left(S_{1}^{2} x\right)+B v\left(S_{1} S_{2} x\right) \\
& =\frac{1}{L}\left[\left(c\left(S_{1} x\right)-c\left(S_{2} x\right)-c\left(S_{1}^{2} x\right)+c\left(S_{1} S_{2} x\right)\right)-0\right. \\
& +\lambda\left(T v\left(S_{3}^{m\left(S_{1} x\right)} S_{1} x\right)-T v\left(S_{3}^{m\left(S_{2} x\right)} S_{2} x\right)-T v\left(S_{3}^{m\left(S_{1}^{2} x\right)} S_{1}^{2} x\right)+T v\left(S_{3}^{m\left(S_{1} S_{2} x\right)} S_{1} S_{2} x\right)\right) 1_{\{n(x) \leq k\}} \\
& +\lambda\left(v\left(S_{3}^{m\left(S_{1} x\right)} S_{1} x\right)-v\left(S_{3}^{m\left(S_{2} x\right)} S_{2} x\right)-v\left(S_{3}^{m\left(S_{1}^{2} x\right)} S_{1}^{2} x\right)+v\left(S_{3}^{m\left(S_{1} S_{2} x\right)} S_{1} S_{2} x\right)\right) 1_{\{n(x)>k\}} \\
& +\mu_{1 i}\left(-v\left(S_{2} x\right) 1_{\{q(x)=0\}}+v(x)-v\left(S_{2} x\right) 1_{\left\{q\left(S_{2} x\right)>0\right\}}-v\left(S_{1} x\right)+v\left(S_{2} x\right)\right) \\
& +\mu_{2 i}\left(T v(x)-T v\left(S_{1} x\right)-v(x) 1_{\left\{q\left(S_{2} x\right) \leq 1\right\}}+v\left(S_{1} x\right) 1_{\left\{q\left(S_{1} S_{2} x\right) \leq 1\right\}}\right. \\
& \left.\left.-T v\left(S_{1}^{-1} x\right) 1_{\left\{q\left(S_{2} x\right)>1\right\}}+T v(x) 1_{\left\{q\left(S_{1} S_{2} x\right)>1\right\}}\right)\right] \leq 0 .
\end{aligned}
$$

The last inequality holds due to the following arguments. The first term in the righthand side of (9) is obviously non-positive according the definition of the immediate cost $c(x)$. Consider the second term with $\lambda$

$$
\begin{aligned}
& \operatorname{Tv}\left(S_{3}^{m\left(S_{1} x\right)} S_{1} x\right)-\operatorname{Tv}\left(S_{3}^{m\left(S_{2} x\right)} S_{2} x\right)-\operatorname{Tv}\left(S_{3}^{m\left(S_{1}^{2} x\right)} S_{1}^{2} x\right)+\operatorname{Tv}\left(S_{3}^{m\left(S_{1} S_{2} x\right)} S_{1} S_{2} x\right) \\
& =\operatorname{Tv}\left(S_{3}^{m\left(S_{1} x\right)} S_{1} x\right)-v\left(S_{3}^{m\left(S_{2} x\right)} S_{1} S_{2} x\right)-\operatorname{Tv}\left(S_{3}^{m\left(S_{1}^{2} x\right)} S_{1}^{2} x\right)+v\left(S_{3}^{m\left(S_{1} S_{2} x\right)} S_{1}^{2} S_{2} x\right) \\
& =\operatorname{Tv}\left(S_{3}^{m(x)} S_{1} x\right)-v\left(S_{3}^{m(x)} S_{1} S_{2} x\right)-\operatorname{Tv}\left(S_{3}^{m(x)} S_{1}^{2} x\right)+v\left(S_{3}^{m(x)} S_{1}^{2} S_{2} x\right),
\end{aligned}
$$

where $m(x)=m\left(S_{1} x\right)=m\left(S_{2} x\right)=m\left(S_{1}^{2} x\right)=m\left(S_{1} S_{2} x\right)$. If the control action $f\left(S_{3}^{m(x)} S_{1}^{2} x\right)=$ $a$ then from the last expression we obtain

$$
\begin{aligned}
& T v\left(S_{3}^{m(x)} S_{1} x\right)-v\left(S_{3}^{m(x)} S_{1} S_{2} x\right)-v\left(S_{3}^{m(x)} S_{1}^{2} S_{a} x\right)+v\left(S_{3}^{m(x)} S_{1}^{2} S_{2} x\right) \\
& \leq v\left(S_{3}^{m(x)} S_{1} S_{a} x\right)-v\left(S_{3}^{m(x)} S_{1} S_{2} x\right)-v\left(S_{3}^{m(x)} S_{1}^{2} S_{a} x\right)+v\left(S_{3}^{m(x)} S_{1}^{2} S_{2} x\right) \leq 0,
\end{aligned}
$$

since for $a=1$ we obtain the inequality (6) in state $S_{3}^{m(x)} x$ and for $a=2$ the term is equal to 0 . The third term is obviously non-positive due to the condition (6) when replacing $x$ by $S_{3}^{m(x)} x$. For the fourth term of (9) we have two cases,

$$
\begin{aligned}
& -v\left(S_{2} x\right) 1_{\{q(x)=0\}}+v(x)-v\left(S_{2} x\right) 1_{\left\{q\left(S_{2} x\right)>0\right\}}-v\left(S_{1} x\right)+v\left(S_{2} x\right) \\
& = \begin{cases}-v\left(S_{2} x\right)+v(x)-v\left(S_{1} x\right)+v\left(S_{2} x\right)=v(x)-v\left(S_{1} x\right) \leq 0 & q(x)=0 \\
v(x)-v\left(S_{2} x\right)-v\left(S_{1} x\right)+v\left(S_{2} x\right)=v(x)-v\left(S_{1} x\right) \leq 0 & q\left(S_{2} x\right)>0 .\end{cases}
\end{aligned}
$$

For the fifth term we have three cases,

$$
\begin{aligned}
& T v(x)-T v\left(S_{1} x\right)-v(x) 1_{\left\{q\left(S_{2} x\right) \leq 1\right\}}+v\left(S_{1} x\right) 1_{\left\{q\left(S_{1} S_{2} x\right) \leq 1\right\}}-T v\left(S_{1}^{-1} x\right) 1_{\left\{q\left(S_{2} x\right)>1\right\}}+T v(x) 1_{\left\{q\left(S_{1} S_{2} x\right)>1\right\}} \\
& = \begin{cases}T v(x)-T v\left(S_{1} x\right)-v(x)+T v(x), & q\left(S_{1} S_{2} x\right) \leq 1, \\
T v(x)-T v\left(S_{1} x\right)-v(x)+v\left(S_{1} x\right), & q\left(S_{2} x\right) \leq 1, q\left(S_{1} S_{2} x\right)>1, \\
T v(x)-T v\left(S_{1} x\right)-T v\left(S_{1}^{-1} x\right)+T v(x), & q\left(S_{2} x\right)>1 .\end{cases}
\end{aligned}
$$

In case $q\left(S_{1} S_{2} x\right) \leq 1$, if we assume $f\left(S_{1} x\right)=1$, then

$$
2 T v(x)-v\left(S_{1}^{2} x\right)-v(x) \leq 2 v\left(S_{1} x\right)-v\left(S_{1}^{2} x\right)-v(x) \leq 0,
$$

due the condition (8), and, if $f\left(S_{1} x\right)=2$, then

$$
2 T v(x)-v\left(S_{1} S_{2} x\right)-v(x) \leq v\left(S_{1} x\right)+v\left(S_{2} x\right)-v\left(S_{1} S_{2} x\right)-v(x) \leq 0
$$


according to (7). In case $q\left(S_{2} x\right) \leq 1, q\left(S_{1} S_{2} x\right)>1$, if $f\left(S_{1} x\right)=1$, then

$$
T v(x)-v\left(S_{1} S_{2} x\right)-v(x)+v\left(S_{1} x\right) \leq v\left(S_{2} x\right)+v\left(S_{1} S_{2} x\right)-v(x)+v\left(S_{1} x\right) \leq 0,
$$

by (7) and if $f\left(S_{1} x\right)=2$, then we get

$$
T v(x)-v\left(S_{1}^{2} x\right)-v(x)+v\left(S_{1} x\right) \leq v\left(S_{1} x\right)-v\left(S_{1}^{2} x\right)-v(x)+v\left(S_{1} x\right) \leq 2 v\left(S_{1} x\right)-v(x)-v\left(S_{1}^{2} x\right) \leq 0
$$

by (8). Finally, in case $q\left(S_{2} x\right)>1$, if $f\left(S_{1} x\right)=1$ and $f\left(S_{1}^{-1} x\right)=1$, then we obtain

$$
T v(x)-v\left(S_{1}^{2} x\right)-v(x)+T v(x) \leq 2 v\left(S_{1} x\right)-v\left(S_{1}^{2} x\right)-v(x) \leq 0,
$$

if $f\left(S_{1} x\right)=2$ and $f\left(S_{1}^{-1} x\right)=1$, then

$$
T v(x)-v\left(S_{1} S_{2} x\right)-v(x)+T v(x) \leq v\left(S_{1} x\right)-v\left(S_{1} S_{2} x\right)-v(x)+v\left(S_{2} x\right) \leq 0,
$$

and, if $f\left(S_{1} x\right)=2$ and $f\left(S_{1}^{-1} x\right)=2$, we have

$$
T v(x)-v\left(S_{1} S_{2} x\right)-v\left(S_{2} S_{1}^{-1} x\right)+T v(x) \leq 2 v\left(S_{2} x\right)-v\left(S_{1} S_{2} x\right)-v\left(S_{2} S_{1}^{-1} x\right) \leq 0,
$$

by condition (8) in state $S_{2} S_{1}^{-1} x$. The case, when $f\left(S_{1} x\right)=1$ and $f\left(S_{1}^{-1} x\right)=2$ is not possible, since it implies that $v\left(S_{1}^{2} x\right) \leq v\left(S_{1} S_{2} x\right)$ and $v\left(S_{2} S_{1}^{-1} x\right) \leq v(x)$. However, from (6) it follows that

$$
v(x)-v\left(S_{2} S_{1}^{-1}\right) \leq v\left(S_{1} x\right)-v\left(S_{2} x\right) \leq v\left(S_{1}^{2} x\right)-v\left(S_{1} S_{2} x\right) \leq 0,
$$

that contradicts to assumption $v\left(S_{2} S_{1}^{-1} x\right) \leq v(x)$. The condition (7) can be proved similarly to the above condition (6). Details are omitted to conserve space.

Proposition 3. For the state $x=(q, 0, n)$ in queue $Q_{i}$ there exists thresholds $q_{2, n, i} \in \mathbb{N}$ for the activation of slower servers such that for the optimal control action $f_{i}^{u(i)}(x)=\operatorname{argmin}_{a \in A(x)} v_{i}\left(S_{a} x\right)$, $i=1,2$, holds

$$
f_{i}^{u(i)}(x)= \begin{cases}2 & d(x)=0, q(x) \geq q_{2, n, i}, \\ 0 & d(x)=0, q(x)<q_{2, n, i} .\end{cases}
$$

Proof. The statement follows directly from a convergence of the policy iteration algorithm $v_{i}^{(k)}(x)=B v_{i}^{(k-1)}$ assuming that $v_{i}^{(0)}(x)=0$ for all $x \in E$, the condition (6) for the function $v(x)=v_{i}^{(0)}(x)$ and from the Proposition 2.

Example 1. Consider the system with an arrival rate $\lambda=20$ and $l=6$ phases of the arrival process. The service rates in $Q_{1}$ are equal to: $\mu_{11}=20, \mu_{21}=4$ and in $Q_{2}: \mu_{12}=10, \mu_{22}=2$. The optimal $k$ for the queue $Q_{1}$ is 4 and $l-k=2$ for the queue $Q_{2}$. The table of optimal control actions $f^{u(i)}(x)$ for the queue $Q_{i}, i=1,2$, are summarized, respectively, in Tables 1 and 2. 
Table 1. Control policy $f_{1}^{k}$ for the queue $Q_{1}$.

\begin{tabular}{cccccccccccccc}
\hline System State $x$ & \multicolumn{11}{c}{ Queue Length $\boldsymbol{q}(x)$} \\
\hline$(d, n)$ & 1 & 2 & 3 & 4 & 5 & 6 & 7 & 8 & 9 & 10 & 11 & 12 & $\ldots$ \\
\hline$(0,1)$ & 0 & 0 & $\mathbf{2}$ & 2 & 2 & 2 & 2 & 2 & 2 & 2 & 2 & 2 & 2 \\
\hline$(0,2)$ & 0 & 0 & $\mathbf{2}$ & 2 & 2 & 2 & 2 & 2 & 2 & 2 & 2 & 2 & 2 \\
\hline$(0,3)$ & 0 & 0 & $\mathbf{2}$ & 2 & 2 & 2 & 2 & 2 & 2 & 2 & 2 & 2 & 2 \\
\hline$(0,4)$ & 0 & 0 & 0 & $\mathbf{2}$ & 2 & 2 & 2 & 2 & 2 & 2 & 2 & 2 & 2 \\
\hline$(0,5)$ & 0 & 0 & 0 & $\mathbf{2}$ & 2 & 2 & 2 & 2 & 2 & 2 & 2 & 2 & 2 \\
\hline$(0,6)$ & 0 & 0 & 0 & $\mathbf{2}$ & 2 & 2 & 2 & 2 & 2 & 2 & 2 & 2 & 2 \\
\hline
\end{tabular}

Table 2. Control policy $f_{2}^{l-k}$ for the queue $Q_{2}$.

\begin{tabular}{cccccccccccccc}
\hline System State $x$ & \multicolumn{110}{c}{ Queue Length $\boldsymbol{q}(\boldsymbol{x})$} \\
\hline$(d, n)$ & 1 & 2 & 3 & 4 & 5 & 6 & 7 & 8 & 9 & 10 & 11 & 12 & $\ldots$ \\
\hline$(0,1)$ & 0 & 0 & $\mathbf{2}$ & 2 & 2 & 2 & 2 & 2 & 2 & 2 & 2 & 2 & 2 \\
\hline$(0,2)$ & 0 & 0 & $\mathbf{2}$ & 2 & 2 & 2 & 2 & 2 & 2 & 2 & 2 & 2 & 2 \\
\hline$(0,3)$ & 0 & 0 & 0 & $\mathbf{2}$ & 2 & 2 & 2 & 2 & 2 & 2 & 2 & 2 & 2 \\
\hline$(0,4)$ & 0 & 0 & 0 & $\mathbf{2}$ & 2 & 2 & 2 & 2 & 2 & 2 & 2 & 2 & 2 \\
\hline$(0,5)$ & 0 & 0 & $\mathbf{2}$ & 2 & 2 & 2 & 2 & 2 & 2 & 2 & 2 & 2 & 2 \\
\hline$(0,6)$ & 0 & 0 & $\mathbf{2}$ & 2 & 2 & 2 & 2 & 2 & 2 & 2 & 2 & 2 & 2 \\
\hline
\end{tabular}

Threshold levels $q_{2, n, 1}=3$ for $n=1,2,3$ and $q_{2, n, 1}=4$ for $n=4,5,6, q_{2, n, 2}=3$ for $n=1,2,5,6$ and $q_{2, n, 2}=4$ for $n=3,4$. In this example $g^{4, f_{1}^{4}, f_{2}^{2}}=2.7962$.

Remark 1. The thresholds $q_{2, n, i}$ for the queue $Q_{i}$ in general are not equal for different arrival phases $n, 1 \leq n \leq 1$. Numerical results show that these thresholds can vary by at most 1 . Hence, we can try to use instead one heuristic threshold level which is independent of $n$. It can be established on the basis of the heuristic solution obtained in [19] for the ordinary queueing system $M / M / K$, where we replace $\lambda$ by a corresponding average arrival rate $\bar{\lambda}_{i}$,

$$
q_{2, i}=\max \left\{2,\left\lfloor\left(\mu_{1 i}-\bar{\lambda}_{i}\right)\left(\frac{1}{\mu_{2 i}}-\frac{1}{\mu_{1 i}}\right)\right\rfloor+2\right\}, i=1,2,
$$

taking into account the fact that the number $q_{2, i}$ here is a sum of customers in the queue and at server 1. An alternative heuristic solution can be obtain using such a solution obtained in [20],

$$
q_{2, i}=\max \left\{2,\left\lfloor\frac{\mu_{1 i}-\bar{\lambda}_{i}+\sqrt{\left(\mu_{1 i}-\bar{\lambda}_{i}\right)^{2}+4 \bar{\lambda}_{i} \mu_{2 i}}}{2 \mu_{2 i}}\right\rfloor+2\right\}, i=1,2
$$

Both of these solutions are of roughly the same quality.

According to the last remark we can reformulate the problem in the following way. Consider the Markov-chain $\left\{X_{i}(t)\right\}_{t \geq 0}$ defined in (2). For the fixed threshold levels $q_{2, i}$ we define a new state-space by

$$
E_{i}=\left\{(q, d, n): 0 \leq q \leq q_{2, i}-1, d \in\{0,1\} \cup q \geq 0, d=1,1 \leq n \leq l\right\} .
$$


This set is partitioned as

$$
\begin{aligned}
& \mathbf{q}=\{(q, 0,1), \ldots,(q, 0, l),(q, 1,1), \ldots,(q, 1, l)\}, 0 \leq q \leq q_{2, i}-1, \\
& \mathbf{q}=\{(q, 1,1), \ldots,(q, 1, l)\}, q \geq q_{2, i} .
\end{aligned}
$$

Proposition 4. The Markov-chain $\left\{X_{i}(t)\right\}_{t \geq 0}$ for the allocation control $f_{0}=u(i)$ is a quasi-birth and death process $(Q B D)$ with a threshold dependent three-diagonal block infinitesimal matrix

$$
\Lambda^{\left(q_{2, i}\right)}=\left(\begin{array}{cccccccccc}
A_{1,0} & A_{0,1} & 0 & 0 & 0 & 0 & 0 & 0 & 0 & \ldots \\
A_{2,0} & A_{1,1} & A_{0,1} & 0 & 0 & 0 & 0 & 0 & 0 & \ldots \\
0 & A_{2,0} & A_{1,1} & A_{0,1} & 0 & 0 & 0 & 0 & 0 & \ldots \\
\ddots & \ddots & \ddots & \ddots & \ddots & \ddots & \ddots & \ddots & \ddots & \ldots \\
0 & \ldots & 0 & A_{2,0} & A_{1,1} & A_{0,1} & 0 & 0 & 0 & \ldots \\
0 & \ldots & 0 & 0 & A_{2,0} & A_{1,2} & A_{0,2} & 0 & 0 & \ldots \\
0 & \ldots & 0 & 0 & 0 & A_{2,1} & A_{1,3} & A_{0,3} & 0 & \ldots \\
0 & \ldots & 0 & 0 & 0 & 0 & A_{2,2} & A_{1,3} & A_{0,3} & \ldots \\
0 & \ldots & 0 & 0 & 0 & 0 & 0 & A_{2,2} & A_{1,3} & \ldots \\
\vdots & \vdots & \vdots & \vdots & \vdots & \vdots & \vdots & \vdots & \vdots & \ddots
\end{array}\right)
$$

Here, the square blocks $A_{1,0}, A_{0,1}, A_{2,0}, A_{1,1}, A_{1,2}$ with dimension $2 l$, the rectangular blocks $A_{2,1}$ and $A_{0,2}$ with dimensions $l \times 2 l$ and $2 l \times l$, and the square blocks $A_{2,2}, A_{1,3}$ and $A_{0,3}$ with dimension $l \times l$ are of the following form

$$
\begin{gathered}
A_{1,0}=\left(\begin{array}{cc}
-\lambda I_{l} & 0 \\
\mu_{2 i} I_{l} & -\left(\lambda+\mu_{2 i}\right) I_{l}
\end{array}\right)+\lambda \sum_{r=u(i)+1}^{l} H_{r, 1+(r \bmod l)} \otimes I_{2} 1_{\{u(i)<l\}}, A_{0,1}=A_{0,3} \otimes I_{2}, \\
A_{2,0}=\mu_{1 i} I_{2 l}, A_{1,1}=A_{1,0}-A_{2,0}, A_{1,2}=A_{1,1}+\left(\begin{array}{cc}
0 & A_{0,3} \\
0 & 0
\end{array}\right), A_{2,1}=\left(0, A_{2,2}\right), A_{2,2}=\left(\mu_{1 i}+\mu_{2 i}\right) I_{l}, \\
A_{0,2}=\left(\begin{array}{c}
0 \\
A_{0,3}
\end{array}\right), A_{1,3}=-\left(\lambda+\mu_{1 i}+\mu_{2 i}\right) I_{l}+\lambda \sum_{r=u(i)+1}^{l} H_{r, 1+(r \bmod l)}, A_{0,3}=\lambda \sum_{r=1}^{u(i)} H_{r, 1+(r \bmod l)} .
\end{gathered}
$$

\section{Proof.}

$$
\begin{aligned}
\lambda \pi_{(0,0, n)} & =\lambda \pi_{(0,0, l)} 1_{\{n=1\}}+\mu_{1 i} \pi_{(1,0,1)}+\mu_{2 i} \pi_{(0,1,1)}, \\
\left(\lambda+\mu_{2 i}\right) \pi_{(0,1, n)} & =\lambda \pi_{(0,1,1)} 1_{\{n=1\}}+\lambda \pi_{(0,1, n-1)} 1_{\{n \geq u(i)+2\}}+\mu_{1 i} \pi_{(1,1, n)}, \\
\left(\lambda+\mu_{1 i}\right) \pi_{(q, 0, n)} & =\lambda \pi_{(q, 0, l)} 1_{\{n=1\}}+\lambda \pi_{(q-1,0, n-1)} 1_{\{2 \leq n \leq u(i)+1\}}+\lambda \pi_{(q, 0, n-1)} 1_{\{n \geq u(i)+2\}} \\
& \mu_{1 i} \pi_{(q+1,0, n)}+\mu_{2 i} \pi_{(q, 1, n)}, 1 \leq q \leq q_{2, i}-1, \\
+\left(\lambda+\mu_{1 i}+\mu_{2 i}\right) \pi_{(q, 1, n)} & =\lambda \pi_{(q, 1, l)} 1_{\{n=1\}}+\lambda \pi_{(q-1,1, n-1)} 1_{\{2 \leq n \leq u(i)+1\}}+\lambda \pi_{(q, 0, n-1)} 1_{\left\{n=q_{2, i}-1\right\}} \\
& +\lambda \pi_{(q, 1, n-1)} 1_{\{n \geq u(i)+2\}}+\mu_{1 i} \pi_{(q+1,1, n)}, 1 \leq q \leq q_{2, i}-1, \\
\left(\lambda+\mu_{1 i}+\mu_{2 i}\right) \pi_{(q, 1, n)} & =\lambda \pi_{(q, 1,1)} 1_{\{n=1\}}+\lambda \pi_{(q-1,1, n-1)} 1_{(2 \leq n \leq u(i)+1)}+\lambda \pi_{(q, 1, n-1)} 1_{\{n \geq u(i)+2\}} \\
& +\left(\mu_{1 i}+\mu_{2 i}\right) \pi_{(q+1,1, n)}, q \geq q_{2, i} .
\end{aligned}
$$

We note that at an arrival epoch in state $(q, 0, n)$ or $(q, 1, n)$ with $q \leq q_{2, i}-1$ the Markov-chain jumps, respectively, to the state $(q+1,0,1+(n \bmod l))$ or $(q+1,1,1+(n$ $\bmod l))$ if $f_{0}(n)=1$ and to the state $(q, 0,1+(n \bmod l))$ or $(q, 1,1+(n \bmod l))$ if $f_{0}(n)=0$. additionally in state $\left(q_{2}-1,0, n\right)$ the transition at the arrival epoch occurs to state $\left(q_{2}-1,1,1+(n \bmod l)\right)$ if $f_{0}(n)=1$. Let $\pi$ partitioned as $\pi_{i}=\left(\pi_{0 i}, \pi_{1 i}, \ldots\right)$ denotes the stationary probability vector of $\Lambda^{\left(q_{2, i}\right)}$, i.e., $\boldsymbol{\pi}_{i} \Lambda^{\left(q_{2, i}\right)}=\mathbf{0}, \boldsymbol{\pi}_{i} \mathbf{e}=1$. By expressing Equation (13) in matrix form we obtain the infinitesimal matrix (12). 
Example 2. In case $l=3$ and $k=2$ for the queues $Q_{1}$ the blocks of the matrix $\Lambda^{\left(q_{2,1}\right)}$ are of the form

$$
\begin{aligned}
& A_{1,0}=\left(\begin{array}{cccccc}
-\lambda & 0 & 0 & 0 & 0 & 0 \\
0 & -\lambda & 0 & 0 & 0 & 0 \\
\lambda & 0 & -\lambda & 0 & 0 & 0 \\
\mu_{2} & 0 & 0 & -\left(\lambda+\mu_{2}\right) & 0 & 0 \\
0 & \mu_{2} & 0 & 0 & -\left(\lambda+\mu_{2}\right) & 0 \\
0 & 0 & \mu_{2} & \lambda & 0 & -\left(\lambda+\mu_{2}\right)
\end{array}\right) \\
& A_{1,1}=\left(\begin{array}{cccccc}
-\left(\lambda+\mu_{1}\right) & 0 & 0 & 0 & 0 & 0 \\
0 & -\left(\lambda+\mu_{1}\right) & 0 & 0 & 0 & 0 \\
\lambda & 0 & -\left(\lambda+\mu_{1}\right) & 0 & 0 & 0 \\
\mu_{2} & 0 & 0 & -\left(\lambda+\mu_{1}+\mu_{2}\right) & 0 & 0 \\
0 & \mu_{2} & 0 & 0 & -\left(\lambda+\mu_{1}+\mu_{2}\right) & 0 \\
0 & 0 & \mu_{2} & \lambda & 0 & -\left(\lambda+\mu_{1}+\mu_{2}\right)
\end{array}\right) \text {, } \\
& A_{1,2}=\left(\begin{array}{cccccc}
-\left(\lambda+\mu_{1}\right) & 0 & 0 & 0 & \lambda & 0 \\
0 & -\left(\lambda+\mu_{1}\right) & 0 & 0 & 0 & \lambda \\
\lambda & 0 & -\left(\lambda+\mu_{1}\right) & 0 & 0 & 0 \\
\mu_{2} & 0 & 0 & -\left(\lambda+\mu_{1}+\mu_{2}\right) & 0 & 0 \\
0 & \mu_{2} & 0 & 0 & -\left(\lambda+\mu_{1}+\mu_{2}\right) & 0 \\
0 & 0 & \mu_{2} & \lambda & 0 & -\left(\lambda+\mu_{1}+\mu_{2}\right)
\end{array}\right), \\
& A_{1,3}=\left(\begin{array}{ccc}
-\left(\lambda+\mu_{1}+\mu_{2}\right) & 0 & 0 \\
0 & -\left(\lambda+\mu_{1}+\mu_{2}\right) & 0 \\
\lambda & 0 & -\left(\lambda+\mu_{1}+\mu_{2}\right)
\end{array}\right) \text {, } \\
& A_{0,1}=\left(\begin{array}{cccccc}
0 & \lambda & 0 & 0 & 0 & 0 \\
0 & 0 & \lambda & 0 & 0 & 0 \\
0 & 0 & 0 & 0 & 0 & 0 \\
0 & 0 & 0 & 0 & \lambda & 0 \\
0 & 0 & 0 & 0 & 0 & \lambda \\
0 & 0 & 0 & 0 & 0 & 0
\end{array}\right) \text {, } \\
& A_{0,2}=\left(\begin{array}{ccc}
0 & 0 & 0 \\
0 & 0 & 0 \\
0 & 0 & 0 \\
0 & \lambda & 0 \\
0 & 0 & \lambda \\
0 & 0 & 0
\end{array}\right), A_{0,3}=\left(\begin{array}{ccc}
0 & \lambda & 0 \\
0 & 0 & \lambda \\
0 & 0 & 0
\end{array}\right) \text {, } \\
& A_{2,0}=\mu_{1} I_{6}, A_{2,1}=\left(\mu_{1}+\mu_{2}\right)\left(\begin{array}{cccccc}
0 & 0 & 0 & 1 & 0 & 0 \\
0 & 0 & 0 & 0 & 1 & 0 \\
0 & 0 & 0 & 0 & 0 & 1
\end{array}\right), A_{2,2}=\left(\mu_{1}+\mu_{2}\right) I_{3} .
\end{aligned}
$$

Proposition 5. The necessary and sufficient condition for the ergodicity of the QBD process $\left\{X_{i}(t)\right\}$ is

$$
\bar{\lambda}_{i}=\frac{u(i)}{l} \lambda<\sum_{j=1}^{2} \mu_{j i}
$$

Proof. For the ergodicity of the the QBD process $\left\{X_{i}(t)\right\}$ according to [21] it is required that $\mathbf{p}_{i} A_{0,3} \mathbf{e}(l)<\mathbf{p}_{i} A_{2,2} \mathbf{e}(l)$, where the stationary probability vector $\mathbf{p}_{i}=\left(p_{1 i}, \ldots, p_{l i}\right)$ satisfies the system $\mathbf{p}\left(A_{0,3}+A_{1,3}+A_{2,2}\right)=0$ and $\mathbf{p}_{i} \mathbf{e}(l)=1$. The matrix $A_{0,3}+A_{1,3}+A_{2,2}=$ $\lambda\left(-I_{l}+\sum_{r=1}^{u}(i) H_{r, r+1}+\sum_{r=u(i)+1}^{l} H_{r, 1+(r \bmod l)}\right)$, which implies that $p_{1 i}=p_{l i}, p_{r-1 i}=$ $p_{r i}, 2 \leq r \leq l$, and hence due to the normalizing condition, $p_{1 i}=\cdots=p_{l i}=\frac{1}{l}$. It is easy to see that $\mathbf{p}_{i} A_{0,3} \mathbf{e}(l)=\frac{u(i)}{l} \lambda$ and $\mathbf{p}_{i} A_{2,2} \mathbf{e}(l)=\mu_{1}+\mu_{2}$. 
If the condition (14) is met, there are stationary state probabilities $\pi_{x}=\lim _{t \rightarrow \infty} \mathbb{P}\left[X_{i}(t)=x\right]$.

Proposition 6. The sub-vectors $\pi_{q i}$ of the stationary state probabilities satisfy the following relations

$$
\begin{aligned}
& \pi_{q i}=\pi_{q_{2, i} i} \prod_{j=1}^{q_{2, i}-q} M_{q_{2, i}-j}, 0 \leq q \leq q_{2, i}-1, \\
& \pi_{q i}=\pi_{q_{2, i} i} R^{q-q_{2, i}}, q \geq q_{2, i},
\end{aligned}
$$

where the matrices $M_{q}$ are defined by

$$
\begin{aligned}
& M_{0}=-A_{2,0} A_{1,0}^{-1} \\
& M_{q}=-A_{2,0}\left(A_{1,1}+M_{q-1} A_{0,1}\right)^{-1}, 1 \leq q \leq q_{2, i}-2, \\
& M_{q_{2, i}-1}=-A_{2,1}\left(A_{1,2}+M_{q_{2, i}-2} A_{0,1}\right)^{-1} .
\end{aligned}
$$

The sub-vector $\pi_{q_{2, i} i}$ is a unique solution of the system

$$
\begin{aligned}
& \boldsymbol{\pi}_{q_{2, i} i}\left(\sum_{q=0}^{q_{2, i}-1} \prod_{j=1}^{q_{2, i}-q} M_{q_{2, i}-j} \mathbf{e}(2 l)+\left(I_{l}-R\right)^{-1} \mathbf{e}(l)\right)=1, \\
& \boldsymbol{\pi}_{q_{2, i} i}\left(A_{1,3}+M_{q_{2, i}-1} A_{0,2}+R A_{2,2}\right)=\mathbf{0} .
\end{aligned}
$$

The matrix $R$ is the minimal non-negative solution to the matrix equation

$$
R^{2} A_{2,2}+R A_{1,3}+A_{0,3}=0 .
$$

Proof. The proof follows directly from the theory of matrix-geometric solutions [21] using a block forward elimination-backward substitution method for the quasi-birth-anddeath processes.

Proposition 7. The mean number of customers $\bar{N}_{i}$ in the queue $Q_{i}$ is given by

$$
\begin{aligned}
\bar{N}_{i}:=\bar{N}_{i}\left(q_{2, i}, u(i), l\right) & =\pi_{q_{2, i} i}\left[\sum_{q=1}^{q_{2, i}-1} q \prod_{j=1}^{q_{2, i}-q} M_{q_{2, i}-j} \mathbf{e}(2 l)+\sum_{q=0}^{q_{2, i}-1} \prod_{j=1}^{q_{2, i}-q} M_{q_{2, i}-j}\left(\sum_{n=l+1}^{2 l} \mathbf{e}_{n}(2 l)\right)\right. \\
& \left.+\left(R\left(I_{l}-R\right)^{-1}+\left(q_{2, i}+1\right) I_{l}\right)\left(I_{l}-R\right)^{-1}\right] .
\end{aligned}
$$

Proof. The mean number of customers in the queueing system $Q_{i}$ satisfies the relation

$$
\bar{N}_{i}=\sum_{q=1}^{q_{2, i}-1} \sum_{n=1}^{2 l} q \pi_{(q, 0, n) i}+\sum_{q=0}^{q_{2, i}-1} \sum_{n=l+1}^{2 l} \pi_{(q, 1, n) i}+\sum_{q=q_{2, i}}^{\infty} \sum_{n=1}^{l}(q+1) \pi_{(q, 1, n) i} .
$$

By expressing relation (20) in vector-matrix form we obtain the expression

$$
\bar{N}_{i}=\sum_{q=1}^{q_{2, i}-1} q \boldsymbol{\pi}_{q i} \mathbf{e}(2 l)+\sum_{q=0}^{q_{2, i}-1} \boldsymbol{\pi}_{q i}\left(\sum_{n=l+1}^{2 l} \mathbf{e}_{n}(2 l)\right)+\boldsymbol{\pi}_{q_{2, i} i} \sum_{q=q_{2, i}}^{\infty}(q+1) R^{q-q_{2, i}},
$$

where the last geometric sum due to the properties of the matrix $R$ converges to the value $\left(R\left(I_{l}-R\right)^{-1}+\left(q_{2, i}+1\right) I_{l}\right)\left(I_{l}-R\right)^{-1}$. The final substitution of (15) to the last relation completes the proof. 
Thus instead of the policy-iteration algorithm we use the results of Propositions 6 and 7 in form of the vector-matrix relation (19) for the mean value

$$
\bar{N}\left(q_{2,1}, q_{2,2}, k, l\right)=\sum_{i=1}^{2} \bar{N}_{i}\left(q_{2, i}, u(i), l\right)
$$

to calculate optimal values $k, l$ and $q_{2, i}, i=1,2$ and provide comparison analysis of an optimal policy with an alternative one.

\section{Bernoulli Splitting Policy}

As an alternative model with a heuristic state independent routing we consider a so-called Bernoulli splitting policy (BSP) model. According to this model, the total arrival rate $\lambda$ is split into rates $p \lambda$ for the system $Q_{1}$ and $(1-p) \lambda$ for the system $Q_{2}$. Obviously, the optimal allocation policy for each parallel queue is of threshold type with corresponding threshold levels $q_{21}$ and $q_{22}$, where $1 \leq q_{21}, q_{22}<\infty$. The optimization problem can be formulated then as follows:

$$
\bar{N}_{1}\left(q_{21}, p \lambda\right)+\bar{N}_{2}\left(q_{22},(1-p \lambda)\right) \Rightarrow \min _{q_{21}, q_{22}, p}
$$

subject to $0 \leq p \leq 1$. First of all, we derive the expression for the mean number of customers in heterogeneous queueing system $M / M / 2$ with an arrival rate $\lambda$ and thresholdbased policy $q_{2}$. This model is obviously a special case of that model discussed in Section 2 for $k=l=1$. The infinitesimal matrix of the Markov-chain describing the queueing system with two heterogeneous servers and threshold-based policy $q_{2}$ is of the same form as in (12), where $A_{1,0}=\left(\begin{array}{cc}-\lambda & 0 \\ \mu_{2} & -\lambda\end{array}\right), A_{1,1}=A_{1,0}-A_{0,2}, A_{0,1}=\lambda I_{2}, A_{0,2}=\mu_{1} I_{2}, A_{1,2}=$ $A_{1,1}+\lambda H_{1,2}, A_{0,2}=\lambda \mathbf{e}_{2}, A_{2,1}=\left(\mu_{1}+\mu_{2}\right) \mathbf{e}_{2}^{\prime}, A_{1,3}=-\left(\lambda+\mu_{1}+\mu_{2}\right), A_{0,3}=\lambda, A_{2,2}=$ $\mu_{1}+\mu_{2}$. To calculate the stationary state probabilities, in principle, one can also use the results of Proposition 6 with $R=\frac{\lambda}{\mu_{1}+\mu_{2}}$. However, for the given two-dimensional process the result can be obtained also directly by solving a system of balance difference equations.

Proposition 8. The stationary probabilities for the threshold policy $q_{2}$ satisfy the relations

$$
\begin{aligned}
& \pi_{(q, 0)}=\left[r_{1}^{q-1-q_{2}}\left(b_{1} \beta_{1}^{q_{2}+1}+b_{2} \beta_{2}^{q_{2}+1}\right)-\left(b_{1} \beta_{1}^{q}+b_{2} \beta_{2}^{q}\right)\right] \pi_{(0,1)}, 0 \leq q \leq q_{2}, \\
& \pi_{(q, 1)}=\left(b_{1} \beta_{1}^{q}+b_{2} \beta_{2}^{q}\right) \pi_{(0,1)}, 0 \leq n \leq q_{2}, \\
& \pi_{(q, 1)}=\rho^{q-q_{2}}\left(b_{1} \beta_{1}^{q_{2}}+b_{2} \beta_{2}^{q_{2}}\right) \pi_{(0,1)}, q \geq q_{2}, \\
& \pi_{(0,1)}=\left[\frac{\rho}{1-\rho}\left(b_{1} \beta_{1}^{q_{2}}+b_{2} \beta_{2}^{q_{2}}\right)+\frac{1-r_{1}^{q_{2}+1}}{\left(1-r_{1}\right) r_{1}^{q_{2}+1}}\left(b_{1} \beta_{1}^{q_{2}+1}+b_{2} \beta_{2}^{q_{2}+1}\right)\right]^{-1},
\end{aligned}
$$

where $\rho=\frac{\lambda}{\mu_{1}+\mu_{2}}$ and $r_{1}=\frac{\lambda}{\mu_{1}}, \beta_{1,2}=\frac{\lambda+\mu_{1}+\mu_{2} \pm \sqrt{\left(\lambda+\mu_{1}+\mu_{2}\right)^{2}-4 \lambda \mu_{1}}}{2 \mu_{1}}, b_{1}=\frac{1-\beta_{1}}{\beta_{2}-\beta_{1}}$ and $b_{2}=$ $\frac{\beta_{2}-1}{\beta_{2}-\beta_{1}}$.

Proof. The system of balance equations is divided into subsystems which can be solved as the system of homogeneous difference equations. For the first subsystem taking into account a threshold policy $q_{2}$ we have

$$
\begin{aligned}
& \left(\lambda+\mu_{2}\right) \pi_{(0,1)}=\mu_{1} \pi_{(1,1)} \\
& \left(\lambda+\mu_{1}+\mu_{2}\right) \pi_{(q, 1)}=\lambda \pi_{(q-1,1)}+\mu_{1} \pi_{(q+1,1)}, 1 \leq q \leq q_{2}-1 .
\end{aligned}
$$

The solution for this system of difference equations can be assumed in form

$$
\pi_{(q, 1)}=\beta^{q} \pi_{(0,1)}, 0 \leq q \leq q_{2} .
$$


By substituting (24) to the difference equations (23) and dividing by $\beta^{q-1}$ we obtain the equation

$$
\mu_{1} \beta^{2}-\left(\lambda+\mu_{1}+\mu_{2}\right) \beta+\lambda=0 .
$$

The last equation has two roots,

$$
\beta_{1,2}=\frac{\left(\lambda+\mu_{1}+\mu_{2} \pm \sqrt{\left(\lambda+\mu_{1}+\mu_{2}\right)^{2}-4 \mu_{1}}\right)}{2 \mu_{1}} .
$$

The probabilities $\pi_{(q, 1)}$ are the solutions of difference equation and they must satisfy the following relation

$$
\pi_{(q, 1)}=\left(b_{1} \beta_{1}^{q}+b_{2} \beta_{2}^{q}\right) \pi_{(0,1)}, 0 \leq q \leq q_{2},
$$

where $b_{1}$ and $b_{2}$ are unknown constant factors. From one side $b_{1}+b_{2}=1$. From the other side, the boundary condition for the probability $\pi_{(0,1)}$ implies

$$
\frac{\lambda+\mu_{2}}{\mu_{1}} \pi_{(0,1)}=\left(\beta_{1}+\beta_{2}-1\right) \pi_{(0,1)}=\left(b_{1} \beta_{1}+b_{2} \beta_{2}\right) \pi_{(0,1)}=\pi_{(1,1)}
$$

and then the expressions for $b_{1}=\frac{1-\beta_{1}}{\beta_{2}-\beta_{1}}$ and $b_{2}=\frac{\beta_{2}-1}{\beta_{2}-\beta_{1}}$. The second subsystem includes equations for the states $x$ with $q(x) \geq q_{2}+1$,

$$
\left(\lambda+\mu_{1}+\mu_{2}\right) \pi_{(q, 1)}=\lambda \pi_{(q-1,1)}+\left(\mu_{1}+\mu_{2}\right) \pi_{(q+1,1)}, q \geq q_{2}+1 .
$$

In this case, a solution of (26) has obviously a geometric form,

$$
\pi_{(q, 1)}=\left(\frac{\lambda}{\mu_{1}+\mu_{2}}\right)^{q-q_{2}} \pi_{\left(q_{2}, 1\right)}=\rho^{q-q_{2}} \pi_{\left(q_{2}, 1\right)}, q \geq q_{2} .
$$

For the third subsystem we obtain,

$$
\begin{aligned}
& \left(\lambda+\mu_{1}\right) \pi_{(q, 0)}=\lambda \pi_{(q-1,0)}+\mu_{1} \pi_{(q+1,0)}+\mu_{2} \pi_{(q, 1)}, 1 \leq q \leq q_{2}-1, \\
& \left(\lambda+\mu_{1}\right) \pi_{\left(q_{2}, 0\right)}=\lambda \pi_{\left(q_{2}-1,1\right)}+\mu_{2} \pi_{\left(q_{2}, 1\right)}, \\
& \left(\lambda+\mu_{1}+\mu_{2}\right) \pi_{\left(q_{2}, 1\right)}=\lambda \pi_{\left(q_{2}-1,1\right)}+\lambda \pi_{\left(q_{2}, 0\right)}+\left(\mu_{1}+\mu_{2}\right) \pi_{\left(q_{2}+1,1\right)} .
\end{aligned}
$$

Now we sum up the Equation (23) for $\pi_{(q, 1)}$ and (28) for $\pi_{(q, 0)}, 1 \leq q \leq q_{2}-1$, and obtain the following difference equations,

$$
\left(\lambda+\mu_{1}\right)\left(\pi_{(q, 0)}+\pi_{(q, 1)}\right)=\lambda\left(\pi_{(q-1,0)}+\pi_{(q-1,1)}\right)+\mu_{1}\left(\pi_{(q+1,0)}+\pi_{(q+1,1)}\right) .
$$

The solution of (29) can be represented in a geometric form

$$
\pi_{(q, 0)}+\pi_{(q, 1)}=\left(\frac{\lambda}{\mu_{1}}\right)^{q}\left(\pi_{(0,0)}+\pi_{(0,1)}\right)=r_{1}^{q}\left(\pi_{(0,0)}+\pi_{(0,1)}\right), 0 \leq q \leq q_{2} .
$$

After some simple algebra we can rewrite (30) in the following way,

$$
\pi_{(q, 0)}=r_{1}^{q-q_{2}}\left(\pi_{\left(q_{2}, 0\right)}+\pi_{\left(q_{2}, 1\right)}\right)-\pi_{(q, 1)}, 0 \leq q \leq q_{2} .
$$


Now, we substitute $\pi_{\left(q_{2}+1,1\right)}=\frac{\lambda}{\mu_{1}+\mu_{2}} \pi_{\left(q_{2}, 1\right)}$ to the last equation of the subsystem (28) and using (25) we obtain

$$
\begin{aligned}
\pi_{\left(q_{2}, 0\right)}+\pi_{\left(q_{2}, 1\right)} & =\frac{\lambda+\mu_{1}+\mu_{2}}{\lambda} \pi_{\left(q_{2}, 1\right)}-\pi_{\left(q_{2}-1,1\right)} \\
& =\frac{\mu_{1}}{\lambda}\left(\frac{\lambda+\mu_{1}+\mu_{2}}{\mu_{1}} \pi_{\left(q_{2}, 1\right)}-\frac{\lambda}{\mu_{1}} \pi_{\left(q_{2}-1,1\right)}\right) \\
& =\frac{\mu_{1}}{\lambda}\left(\left(\beta_{1}+\beta_{2}\right) \pi_{\left(q_{2}, 1\right)}-\beta_{1} \beta_{2} \pi_{\left(q_{2}-1,1\right)}\right) \\
& =r_{1}^{-1}\left(b_{1} \beta_{1}^{q_{2}+1}+b_{2} \beta_{2}^{q_{2}+1}\right) \pi_{(0,1)} .
\end{aligned}
$$

Combination of (31) with (32) leads to expression (22) for $\pi_{(q, 0)}$. Finally, the probability $\pi_{(0,1)}$ is calculated from the normalizing condition $\sum_{q=0}^{q_{2}} \pi_{(q, 0)}+\sum_{q=0}^{\infty} \pi_{(q, 1)}=1$.

Proposition 9. The mean number of customers $\bar{N}$ is of the form

$$
\begin{aligned}
\bar{N} & :=\bar{N}\left(q_{2}, \lambda\right)=\left[b_{1} \frac{1+\beta_{1}^{q_{2}+1} q_{2}-\beta_{1}^{q_{2}}\left(1+q_{2}\right)}{1-\beta_{1}}+b_{2} \frac{1+\beta_{2}^{q_{2}+1} q_{2}-\beta_{2}^{q_{2}}\left(1+q_{2}\right)}{1-\beta_{2}}\right. \\
& \left.+\frac{r_{1}\left(1-\left(1+q_{2}\right) r_{1}^{q_{2}}+q_{2} r_{1}^{q_{2}+1}\right)}{r_{1}^{q_{2}+1}\left(1-r_{1}\right)^{2}}\left(b_{1} \beta_{1}^{q_{2}+1}+b_{2} \beta_{2}^{q_{2}+1}\right)+\frac{1+q_{2}-q_{2} \rho}{(1-\rho)^{2}}\left(b_{1} \beta_{1}^{q_{2}}+b_{2} \beta_{2}^{q_{2}}\right)\right] \\
& \times\left[\frac{\rho}{1-\rho}\left(b_{1} \beta_{1}^{q_{2}}+b_{2} \beta_{2}^{q_{2}}\right)+\frac{1-r_{1}^{q_{2}+1}}{\left(1-r_{1}\right) r_{1}^{q_{2}+1}}\left(b_{1} \beta_{1}^{q_{2}+1}+b_{2} \beta_{2}^{q_{2}+1}\right)\right]^{-1}
\end{aligned}
$$

Proof. For the mean number of customers in the system under the threshold policy $q_{2}$ we obtain

$$
\bar{N}\left(q_{2}, \lambda\right)=\sum_{q=0}^{q_{2}} q \pi_{(q, 0)}+\sum_{q=0}^{q_{2}-1}(q+1) \pi_{(q, 1)}+\sum_{q=q_{2}}^{\infty}(q+1) \pi_{(q, 1)} .
$$

By substituting expressions (22) after some algebra for geometric series we obtain the relation (33).

The results of Propositions 8 and 9 are characteristic for the Bernoulli splitting policy. Using (33) we solve optimization problem (21). As a heuristic solution for the threshold $q_{2}$ we use the solutions obtained by (10) and (11) for the optimal open-loop policy. For numerical experiments we replace in these expressions $\bar{\lambda}_{i}$ by $\lambda((1-2 p)(i-1)+p)$ and then optimized numerically the functional

$$
\bar{N}\left(q_{2,1}, q_{2,2}, p\right)=\sum_{i=1}^{2} \bar{N}_{i}\left(q_{2, i},((1-2 p)(i-1)+p) \lambda\right) \Rightarrow \min _{q_{2,1}, q_{2,2}, p} .
$$

\section{Numerical Analysis}

In this section, we provide numerical examples which include a comparative analysis of calculations obtained by means of the policy iteration algorithm, the matrix-analytical method, and expressions obtained for the Bernoulli splitting model.

In a first example we compare the results on the behavior of $\bar{N}^{f}$ calculated numerically for the optimal threshold policy (OTP) where threshold levels depend on the phase of the arrival process and using analytic vector-matrix relations for the heuristic threshold policy (HTP) defined in (10). The arrival intensity $\lambda$ is fixed at $\lambda=20$ and the number of arrival phases $l=6$. The Router 0 dispatches the customers according to the optimal open-loop control (OLC) with value $k$. We fix also heterogeneity factors for the queues $Q_{1}$ and $Q_{2}$ at level $\mu_{11} / \mu_{21}=\mu_{12} / \mu_{22}=5$. The following cases are under study:

- $\quad$ Case 1: $\mu_{11}=15, \mu_{21}=3$; 
- $\quad$ Case 2: $\mu_{11}=20, \mu_{21}=4$;

- $\quad$ Case 3: $\mu_{11}=25, \mu_{21}=5$;

- $\quad$ Case 4: $\mu_{11}=30, \mu_{21}=6$.

Note that the main conclusions about behavior of the average number of customers in the system drawn from the proposed experiments at given cases are also valid for other values of service intensities and heterogeneity factors, provided that the total intensity in queue $Q_{1}$ is higher than in queue $Q_{2}$.

(a)

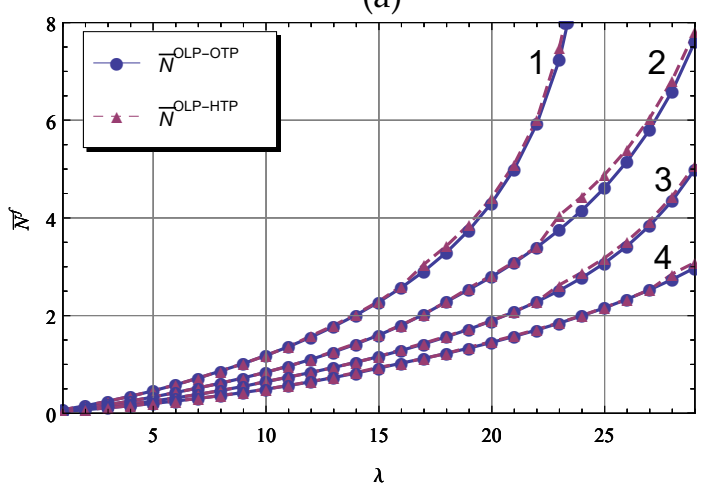

(b)

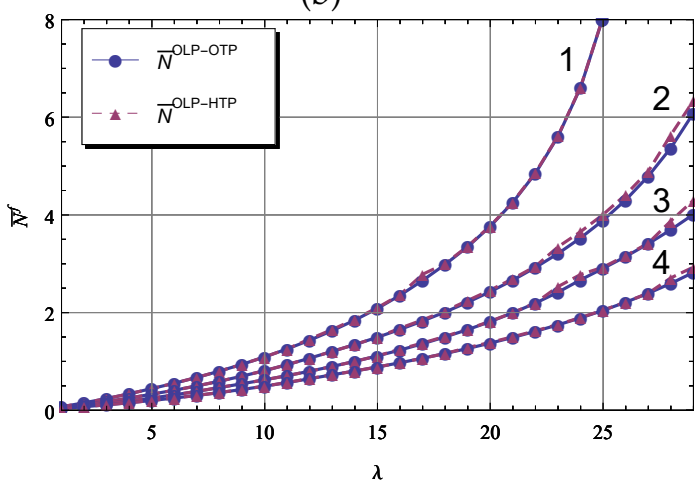

Figure 2. $\bar{N}^{f}$ for $\mu_{12}=10, \mu_{22}=2$ (a) and for $\mu_{12}=12, \mu_{22}=2.4(\mathbf{b})$ versus $\lambda, \mu_{11}$ and $\mu_{21}$.

In Figure 2, we display the mean values $\bar{N}^{\mathrm{OLP}-\mathrm{OTP}}$ and $\bar{N}^{\mathrm{OLP}-H T P}$ as $\lambda$ varies in the interval $[1,29]$, the service rates $\mu_{11}$ and $\mu_{21}$ of the queue $Q_{1}$ vary according to specified cases, for the queue $Q_{2}$ we set $\mu_{12}=10, \mu_{22}=2$ in a figure labeled by (a) and $\mu_{12}=12, \mu_{22}=2.4$ in a figure labeled by (b). As to be expected, the total mean number of customers in the system increases monotonously with increasing values of the traffic intensity and decreasing of service rates. The curves in most cases are graphically indistinguishable in the displayed domain. Only in some cases of high load there is a slight difference in the curves and in general the optimal policy has a very little advantage over the heuristic one. Thus, for different system parameters the influence of individual states of the arrival process can be neglected, so that the Routers 1 and 2 needs only the information $q_{2, i}$.

The optimal open-loop control policy $k$ for different system parameters are illustrated the following tables based on the results of the previous experiment. In Table 3, we take for the queue $Q_{2}$ the service rates $\mu_{12}=10$ and $\mu_{22}=2$ and, in Table 4 , the service rates of $Q_{2}$ are equal to $\mu_{12}=12$ and $\mu_{22}=2$.4. The maximal possible number of arrival phases is as before fixed to $l=6$. From these Tables, we may remark that in light-traffic case, e.g., when $\lambda<\mu_{11}-\mu_{12}$, the Router 0 uses only the queue $Q_{1}$ and the optimal policy $k=l=6$. As $\lambda$ increases, the incentive to make an assignment to the slower queue $Q_{2}$ is rising higher. When the difference in service rate between the two queues decreases, Router 0 starts sending more customers to the slower queue $Q_{2}$ at lower traffic intensity values. For example, if $\mu_{11}=15, \mu_{21}=3$, in a heavy traffic case, $\lambda>17$, the optimal policy is equal to $k=4$ when $\mu_{12}=10, \mu_{22}=2$, i.e., only $1 / 3$ of customers must be sent to $Q_{2}$, while $k=3$ when $\mu_{12}=12, \mu_{22}=2.4$, i.e., $1 / 2$ of customers must be dispatched to queue $Q_{2}$.

Table 3. Optimal policy $k$ versus $\lambda, \mu_{11}$ and $\mu_{21}$ for fixed $\mu_{12}=10, \mu_{22}=2$.

\begin{tabular}{cccccc}
\hline$\left(\mu_{11}, \mu_{21}\right)$ & $\lambda$ & $\boldsymbol{k}$ & $\left(\mu_{11}, \mu_{\mathbf{2 1}}\right)$ & $\boldsymbol{\lambda}$ & $\boldsymbol{k}$ \\
\hline \multirow{3}{*}{$(15,3)$} & {$[1,2]$} & 6 & & {$[1,6]$} & 6 \\
& {$[3,7]$} & 5 & $(20,4)$ & {$[7,18]$} & 5 \\
& {$[8,29]$} & 4 & & {$[19,29]$} & 4 \\
\hline \multirow{2}{*}{$(25,5)$} & {$[1,10]$} & 6 & \multirow{2}{*}{$(30,6)$} & {$[1,15]$} & 6 \\
& {$[11,29]$} & 5 & & {$[16,29]$} & 5 \\
\hline
\end{tabular}


Table 4. Optimal policy $k$ versus $\lambda, \mu_{11}$ and $\mu_{21}$ for fixed $\mu_{12}=12, \mu_{22}=2.4$

\begin{tabular}{cccccc}
\hline$\left(\mu_{11}, \mu_{21}\right)$ & $\lambda$ & $k$ & $\left(\mu_{11}, \mu_{21}\right)$ & $\lambda$ & $k$ \\
\hline \multirow{3}{*}{$(15,3)$} & 1 & 6 & & {$[1,4]$} & 6 \\
& {$[2,4]$} & 5 & $(20,4)$ & {$[5,12]$} & 5 \\
& {$[5,17]$} & 4 & & {$[13,29]$} & 4 \\
\hline \multirow{3}{*}{$(25,5)$} & {$[18,29]$} & 3 & & & \\
& {$[1,8]$} & 6 & & {$[1,12]$} & 6 \\
& {$[9,24]$} & 5 & $(30,6)$ & {$[13,29]$} & 5 \\
\hline
\end{tabular}

It is interesting to show the influence of the number of the phases $l$ of the arrival process. This is done in Figures 3 and 4, respectively, for the queue $Q_{1}$ and $Q_{2}$, where in figures labeled by (a) we illustrate the mean number of customers in each queue operating under the HTP and by (b) a 1-lag correlation with $l=3,6,9$. The arrival rate varies in the interval [1,29], the service rates in $Q_{1}$ and $Q_{2}$ are fixed at $\mu_{11}=20, \mu_{21}=4$, and $\mu_{12}=10, \mu_{22}=2$. We observe that the mean number of customers $\bar{N}_{i}^{\text {OLP-HTP }}$ in each queue $i$ changes with jumps as $\lambda$ increases. Jumps occur where policy $k$ changes, as can be clearly seen by the behavior of the correlation function $r_{i}$. Moreover, the function $\bar{N}_{1}^{\text {OLP-HTP }}$ increases non-monotonically, although the function $\bar{N}^{\mathrm{OLP}-\mathrm{HTP}}=\overline{\mathrm{N}}_{1}^{\mathrm{OLP}-\mathrm{HTP}}+\overline{\mathrm{N}}_{2}^{\mathrm{OLP}-\mathrm{HTP}}$ has due to previous experiments a monotonic structure by increasing $\lambda$ and the choice $l=3$ for the original system and specified service rates seems to be the better one.

We can also compare the performance of the optimal open-loop policy (OLP) $k$ and the Bernoulli-splitting policy (BSP). For the allocation of customers between the servers the heuristic threshold policy (HTP) is chosen. In Figure 5, we consider the mean values $\bar{N}^{f}$ when $\lambda$ increases, service rates of $Q_{1}$ change according to specified above cases, service rates of $Q_{2}$ are equal to $\mu_{12}=10, \mu_{22}=2$ in a figure labeled by (a) and $\mu_{12}=12, \mu_{22}=2.4$ in a figure labeled by (b). We see that the OLP outperforms the BSP despite the fact that under the OLP the arrival stream has correlated inter-arrival times, and as it is known, correlation in Markovian arrival processes often leads to a significant increase in the mean number of customers in the system. such an advantage is drastically reduced in a lighttraffic and heavy-traffic cases. The advantage of the OLP in performance metric $\bar{N}^{f}$ can be up to more than $25 \%$ for certain values of system parameter values compared to the heuristic BSP.

(a)

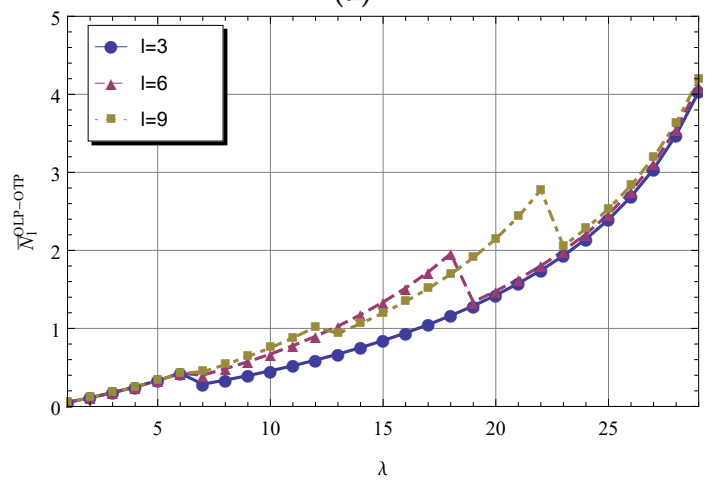

(b)

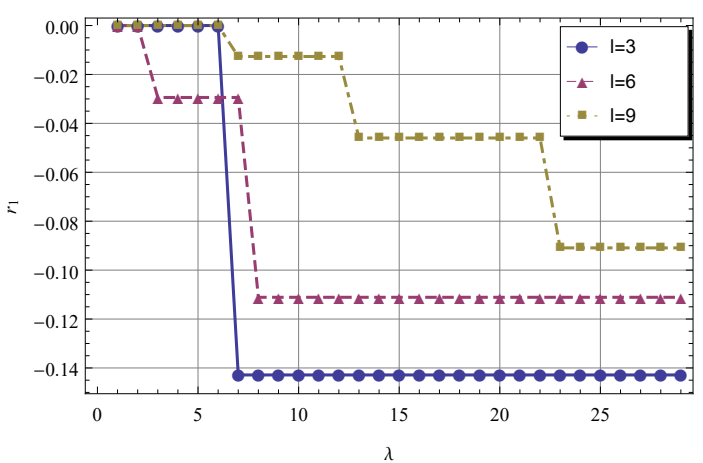

Figure 3. $\bar{N}_{1}^{f}$ (a) and $r_{1}(\mathbf{b})$ versus $\lambda$ and $l$. 
(a)

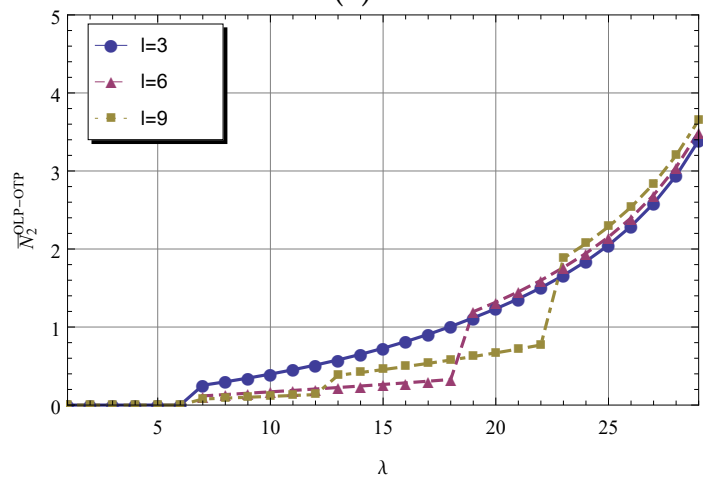

(b)

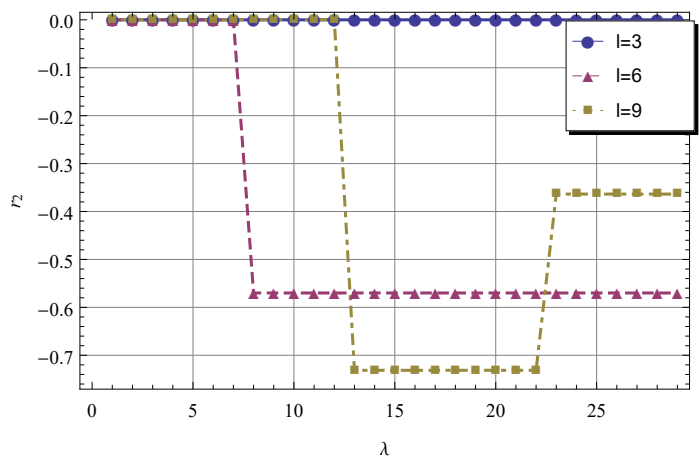

Figure 4. $\bar{N}_{2}^{f}(\mathbf{a})$ and $r_{2}(\mathbf{b})$ versus $\lambda$ and $l$.

(a)

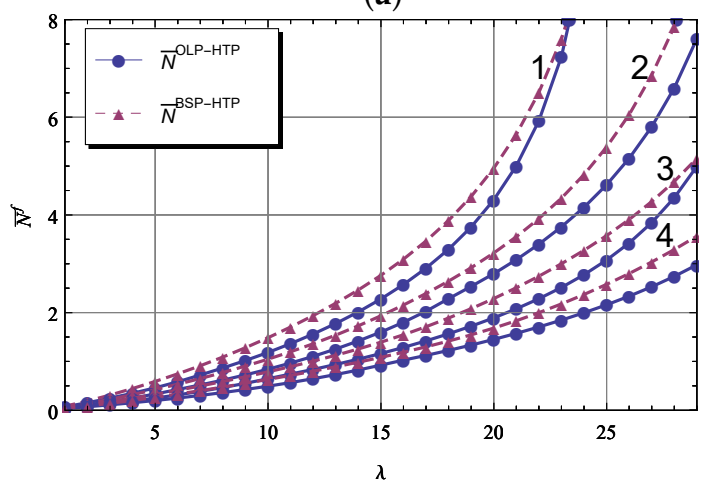

(b)

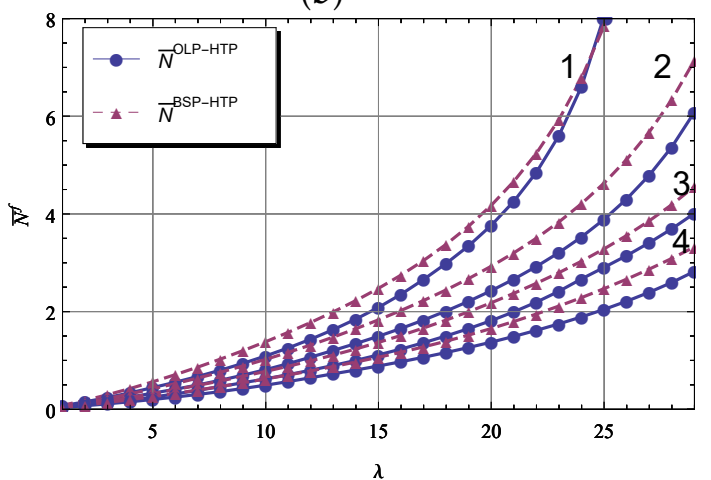

Figure 5. Functions $\bar{N}^{f}$ for $\mu_{12}=10, \mu_{22}=2$ (a) and for $\mu_{12}=12, \mu_{22}=2.4$ (b) versus $\lambda, \mu_{11}$ and $\mu_{21}$.

\section{Conclusions}

In this research, we provide the model to calculate the optimal open-loop routing policy and corresponding optimal allocation policies for the servers in two heterogeneous queues operating in parallel. It is show that the routing policy influences the arrival process of the specific queue. It is reflected in the fact that the optimal threshold policy to dispatch the customers between servers depends both on the number of waiting customers and on the phase of the arrival process. For the heuristic threshold policy a matrix-analytic solution is obtained. The quality of such a solution is compared with an alternative static policy defined by the optimal Bernoulli splitting. The optimal open-loop policy outperforms the probabilistic heuristic policy and advantage in a mean sojourn time can be more than $25 \%$. This topic can be developed further to provide an exhaustive performance analysis, including not only the calculation of average characteristics, but also stationary waiting time distributions for a given control policy. A comparison of the proposed routing policy with an optimal dynamic closed-loop policy is also a topic for subsequent research.

Author Contributions: Conceptualization, D.E.; formal analysis, investigation, methodology, software and writing, D.E. and N.S. All authors have read and agreed to the published version of the manuscript.

Funding: Open Access Funding by the Johannes Kepler University Linz. This research has been supported by the RUDN University Strategic Academic Leadership Program (recipient D. Efrosinin).

Institutional Review Board Statement: Not applicable.

Informed Consent Statement: Not applicable.

Data Availability Statement: Not applicable. 
Acknowledgments: This paper has been supported by the RUDN University Strategic Academic Leadership Program (recipient D. Efrosinin) and Open Access Funding by the Johannes Kepler University Linz.

Conflicts of Interest: The authors declare no conflict of interest.

\section{References}

1. Koole, G. Routing to parallel homogeneous queues. Math. Methods Oper. Res. 2005, 1-4. [CrossRef]

2. Hordijk, A.; Koole, G. On the assignment of customers to parallel queues. Probab. Eng. Inf. Sci. 1992, 6, 495-511. [CrossRef]

3. Koyanagi, J.; Kawai, H. An assignment problem for a parallel queueing system with two heterogeneous servers. Math. Comput. Model. 1995, 22, 173-181. [CrossRef]

4. Delasay, M.; Kolfal, B.; Ingolfsson, A. Maximizing throughput in finite-source parallel queue systems. Eur. J. Oper. Res. 2012, 217, 554-559. [CrossRef]

5. Iravani, S.M.R.; Krishnamurthy, V.; Chao, G.H. Optimal server scheduling in nonpreemptive finite-population queueing system. Queueing Syst. 2007, 55, 95-105. [CrossRef]

6. Hyytiä, E.; Righter, R.; Samuelsson, S.G. Beyond Shortest Queue Routing with Heterogeneous Servers and General Cost Functions; Valuetools: Venice, Italy, 2017.

7. Niño-Mora, J. Resource allocation and routing in parallel multi-server queues with abandonments for cloud profit maximization. Comput. Oper. Res. 2019, 103, 221-236. [CrossRef]

8. Milito, R.A.; Fernández-Gaucherand, E. Open-loop routing of $N$ arrivals to $M$ parallel queues. IEEE Trans. Autom. Control 1995, 40, 2108-2114. [CrossRef]

9. Gaujal, B.; Hyon, E.; Jean-Marie, A. Optimal Routing in Two Parallel Queues with Exponential Service Times. Discrete Event Dyn. Syst. 2006, 16, 71-107. [CrossRef]

10. Koole, G. A simple proof of the optimality of a threshold policy in a two-server queueing system. Syst. Control Lett. 1995, 26, 301-303. [CrossRef]

11. Lin, W.; Kumar, P.R. Optimal control of a queueing system with two heterogeneous servers. IEEE Trans. Autom. Control 1984, 29, 696-703. [CrossRef]

12. Özkan, E.; Kharoufeh, J.P. Optimal control of a two-server queueing system with failures. Probab. Eng. Inf. Sci. 2014, $28,489-527$. [CrossRef]

13. Rykov, V.; Efrosinin, D. On the slow server problem. Autom. Remote Control 2010, 70, 2013-2023. [CrossRef]

14. Boel, R.K.; Talat, N.K. Performance Analysis and Optimal Threshold Policies for Queueing Systems with Several Heterogeneous Servers and Markov-Modulated Arrivals. Matrix-Anal. Methods Stoch. Models 1997, 183, 117-136.

15. Efrosinin, D. Controlled Queueing Systems with Heterogeneous Servers: Dynamic Optimization and Monotonicity Properties of Optimal Control Policies in Multiserver Heterogeneous Queues; VDM Verlag: Saarbrücken, Germany, 2008

16. Puterman, M.L. Markov Decision Processes: Discrete Stochastic DYNAMIC programming; John Wiley \& Sons, Inc.: Hoboken, NJ, USA, 1994.

17. Howard, R.A. Dynamic Programming and Markov Processes; Wiley Series: Hoboken, NJ, USA, 1960.

18. Sennott, L.I. Average cost optimal stationary policies in infinite state Markov decision processes with unbounded costs. Oper. Res. 1989, 37, 626-633. [CrossRef]

19. Efrosinin, D.; Stepanova, N. Estimation of the optimal threshold policy in a queue with heterogeneous servers Using a heuristic solution and artificial neural networks. Mathematics 2021, 9, 1267. [CrossRef]

20. Efrosinin, D.; Rykov, V. Heuristic solution for the optimal thresholds in a controllable multi-server heterogeneous queueing system without preemption. In Distributed Computer and Communication Networks. DCCN 2015. Communications in Computer and Information Science; Vishnevsky, V., Kozyrev, D., Eds.; Springer: Berlin/Heidelberg, Germany, 2016; Volume 601, pp. $238-252$.

21. Neuts, M.F. Matrix-Geometric Solutions in Stochastic Models; The John Hopkins Univ. Press: Baltimore, MD, USA, 1981. 